\title{
Dead Ends Limit Charge Carrier Extraction from All-Polymer Bulk Heterojunction Solar Cells
}

Rokas J asiunas, Armantas Melianas, Yuxin Xia, Nikolaos Felekidis, Vidmantas Gulbinas and Martijn Kemerink

The self-archived postprint version of this journal article is available at Linköping University Institutional Repository (DiVA):

http:/ / urn.kb.se/ resolve?urn=urn:nbn:se:liu:diva-150877

N.B.: When citing this work, cite the original publication.

J asiunas, R., Melianas, A., Xia, Y., Felekidis, N., Gulbinas, V., Kemerink, M., (2018), Dead Ends Limit Charge Carrier Extraction from All-Polymer Bulk Heterojunction Solar Cells, ADVANCED

ELECTRONIC MATERIALS, 4(8), 1800144. https:// doi.org/ 10.1002/aelm.201800144

Original publication available at:

https:// doi.org/ 10.1002/ aelm.201800144

Copyright: Wiley (12 months)

http:// eu.wiley.com/WileyCDA/ 


\section{WILEY-VCH}

DOI: $10.1002 /$

Article type: Full Paper

\section{Dead ends limit charge carrier extraction from all-polymer bulk heterojunction solar cells}

Rokas Jasiūnas*, Armantas Melianas, Yuxin Xia, Nikolaos Felekidis, Vidmantas Gulbinas and Martijn Kemerink

Rokas Jasiūnas, Prof. Vidmantas Gulbinas

Center for Physical Sciences and Technology, Saulėtekio av. 3, LT-10257, Vilnius, Lithuania

E-mail: rokas.jasiunas@ftmc.lt

Dr. Armantas Melianas, Yuxin Xia

Biomolecular and Organic Electronics, Department of Physics, Chemistry and Biology (IFM), Linköping University, 58183 Linköping, Sweden

Dr. Armantas Melianas

Present address: Department of Materials Science and Engineering, Stanford University, Stanford, California 94305, USA

Nikolaos Felekidis, Prof. Martijn Kemerink

Complex Materials and Devices, Department of Physics, Chemistry and Biology (IFM),

Linköping University, 58183 Linköping, Sweden

Keywords: organic photovoltaic, all-polymer, spatial traps

Extraction of photocreated charge carriers from a prototypical all-polymer organic solar cell is investigated by combining transient photocurrent and time-delayed collection field experiments with numerical simulations. It is found that extraction is significantly hampered by charges getting trapped in spatial traps that are tentatively attributed to dead ends in the intermixed polymer network - in photovoltaic devices based on the same donor polymer and a fullerene acceptor this effect is much weaker. The slow-down in charge extraction leads to enhanced recombination and associated performance losses. These effects are observed in addition to the dispersive behavior that is characteristic of charge motion in energetically disordered media. Upon annealing the effects of spatial traps diminish, rationalizing the doubling in device power conversion efficiency after annealing.

\section{Introduction}




\section{WILEY-VCH}

Extraction of photogenerated charge carriers from organic bulk heterojunction (BHJ) solar cells is one of the most important processes determining device performance. Slow carrier extraction causes their accumulation in the active layer and, as a consequence, an increased bimolecular recombination, which reduces the short-circuit current and particularly the fill factor. ${ }^{[1]}$ It is commonly assumed that the optimal morphology for efficient BHJ solar cells is one where photogenerated electrons and holes avoid recombination by moving via spatially separated percolating domains consisting (predominantly) of electron accepting material in one phase and donating material in the other. ${ }^{[2]}$ However, such strongly phase separated morphologies are prone to cause extraction problems due to the formation of dead ends and/or isolated domains. $^{[3]}$

Fortunately, in state of the art polymer:fullerene and polymer:small molecule organic photovoltaic (OPV) devices performance is not limited by recombination losses during extraction (but mostly by voltage losses) as witnessed by large fill factors and relatively high internal quantum efficiency (IQE) values. ${ }^{[4]}$ As a consequence, extraction of photocreated charge carriers can often be well described using models that consider the BHJ as an effective medium with the energy levels of the medium reflecting the HOMO of the donor and the LUMO of the acceptor. ${ }^{[5,6]}$ In such models, morphological effects are only implicitly accounted for by the values of the (effective) transport and disorder parameters. One may wonder why this evident simplification works so well. It has recently become clear that one important reason might be that charge carriers in organic semiconductors may actually be able to move over relatively large distances, up to several $\mathrm{nm}$, by long-range tunneling or molecular superexchange. ${ }^{[7-10]}$ This enables transport to non-nearest neighbor sites, and thereby greatly relaxes the need to have connected phases of pure material for efficient charge transport.

OPV devices using non-fullerene acceptors are currently receiving massive attention and start to outperform fullerene-based devices. ${ }^{[11-13]}$ While having evident benefits over fullerenes in 


\section{WILEY-VCH}

terms of improved absorption and larger energy level tunability, charge transport and extraction in non-fullerene BHJ is still less understood. In particular all-polymer BHJ, in which both donor and acceptor materials are polymers, are in many respects in their infancy. While the entanglement of polymer chains in such BHJ is expected to lead to a much-needed increased morphological stability at elevated temperatures inherent to solar cell operation, ${ }^{[14]}$ the same entanglement can also lead to extraction problems. Such problems are less pronounced in BHJ where at least one of the constituents is a small molecule where dispersion of a minor fraction of the small molecule in the other compound is sufficient to enable reasonably efficient charge transport between these molecules. ${ }^{[7,9]}$ However, the required 'molecular' dispersion, in which sites of a given material are located within a few nm one from another is much harder to realize with polymer chains containing a significant number of repeat units and long side chains, rendering a large fraction of the volume insulating. In allpolymer OPV one may therefore anticipate additional transport problems associated with isolated polymer clusters or polymer chains forming dead ends.

An analogous situation has been observed for 1,1-bis[(di-4-tolylamino)phenyl]cyclohexane (TAPC) in a polycarbonate matrix, where the carrier mobility has been found to decrease with increasing electric field strength. ${ }^{[15]}$ This was attributed to dead ends where carriers need to hop against the direction of the field to escape the spatial trap and continue drift. ${ }^{[16]}$

Here, we combine transient charge extraction experiments with numerical modelling to demonstrate that in a prototypical all-polymer OPV device, consisting of TQ1 as the donor and N2200 as the acceptor, spatial traps indeed lead to charge extraction problems and, concomitantly, to increased recombination. Upon annealing the effect of these spatial traps largely vanishes. We have previously shown that annealing roughly doubles the device's power conversion efficiency while, surprisingly, the ps- $\mu$ s charge extraction kinetics were unaffected. We tentatively associate spatial traps with chain ends that hamper the initial, short-range separation of the photocreated electron-hole pair, leading to strongly enhanced 


\section{WILEY-VCH}

geminate recombination. ${ }^{[17]}$ In a broader perspective, these results highlight a morphological problem which may occur in all bulk-heterojunction OPVs, and show that relatively simple processing steps, such as thermal annealing, can possibly at least partly resolve the issue.

\section{Results}

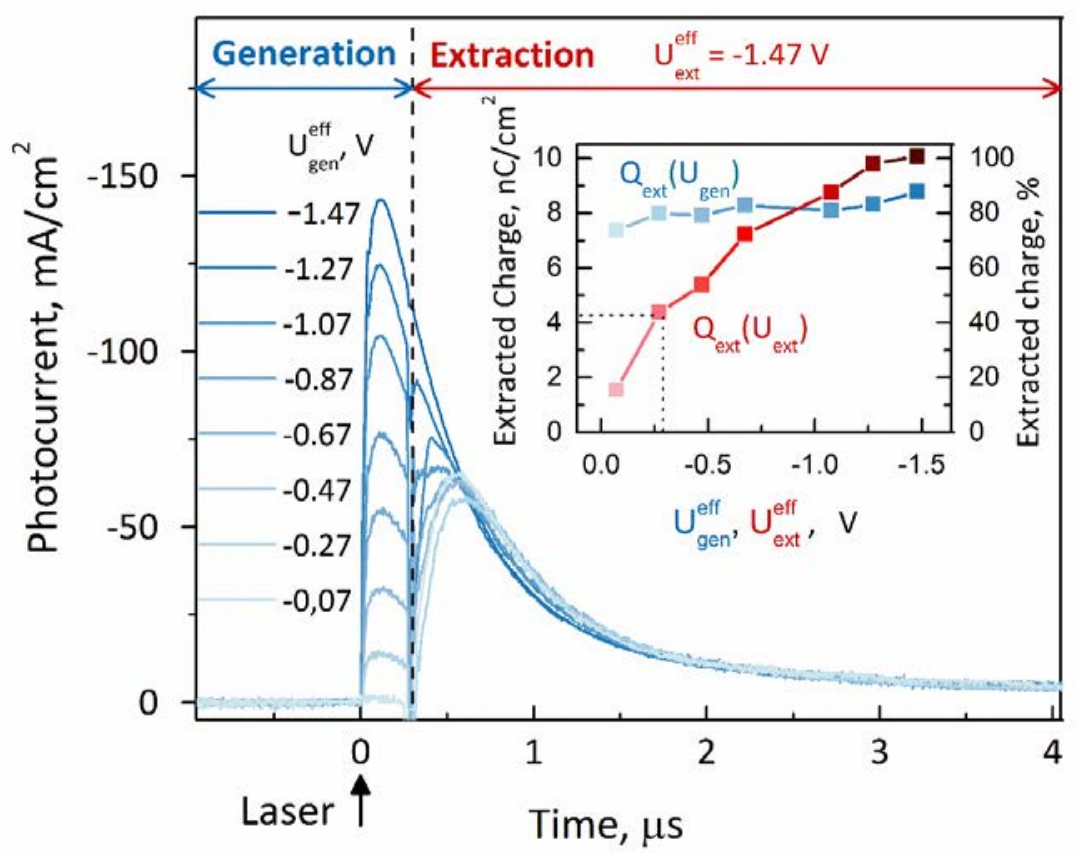

Figure 1. TDCF photocurrent kinetics for the as-cast sample at different effective generation voltages measured with a constant effective collection voltage $U^{\text {eff }}=-1.47 \mathrm{~V}$ at a delay time of $0.3 \mu \mathrm{s}$. The inset shows the total charge extracted vs. generation voltage at $U_{\text {ext }}^{\text {eff }}=-1.47 \mathrm{~V}$ (blue line + symbols) and vs. extraction voltage at zero effective generation voltage, i.e. $U_{\text {appl }}$ $=-\mathrm{U}_{\mathrm{Bl}}=0.47 \mathrm{~V}$ (red line + symbols). The black dashed line is a guide to the eye.

Figure 1 shows the TDCF investigation results obtained for the as-spun TQ1:N2200 sample with a constant extraction (collection) delay of 300 ns but at different generation voltages $\mathrm{U}_{\text {gen. }}$. Note that all voltages specified here and below are effective values that account for the applied voltage and the built-in potential $\mathrm{U}^{\text {eff }}=\mathrm{U}_{\mathrm{appl}}+\mathrm{V}_{\mathrm{BI}}\left(\mathrm{V}_{\mathrm{Bl}}=-0.47 \mathrm{~V}\right)$. The effective extraction voltage was kept constant at $U_{\text {ext }}=-1.47 \mathrm{~V}$, where the minus sign corresponds to reverse bias. The delay time of 300 ns was chosen to make sure that the specified collection field was indeed fully applied at this time. Similar measurements were also performed at close 


\section{WILEY-VCH}

to zero effective generation voltage $\left(\mathrm{U}_{\mathrm{appl}}-\mathrm{V}_{\mathrm{Bl}}\right)=0 \mathrm{~V}$ by changing the collection voltage, yielding consistent results (kinetics not shown). The inset in Figure 1 summarizes the results, showing the dependence of the total extracted charge versus the generation and collection voltages. The strong dependence of the collected charge on the collection voltage is consistent with the modest fill factor of the as-cast devices. ${ }^{[18]}$ The dependence on the generation voltage suggests that the carrier generation efficiency is dependent on the electric field, which would indicate a charge generation problem due to geminate recombination. In fact, in a previous work we used a detailed analysis of the recombination kinetics on the ps- $\mu$ s timescale to identify field-dependent geminate recombination as the major loss channel in the as-cast devices compared to the annealed ones. ${ }^{[17]}$

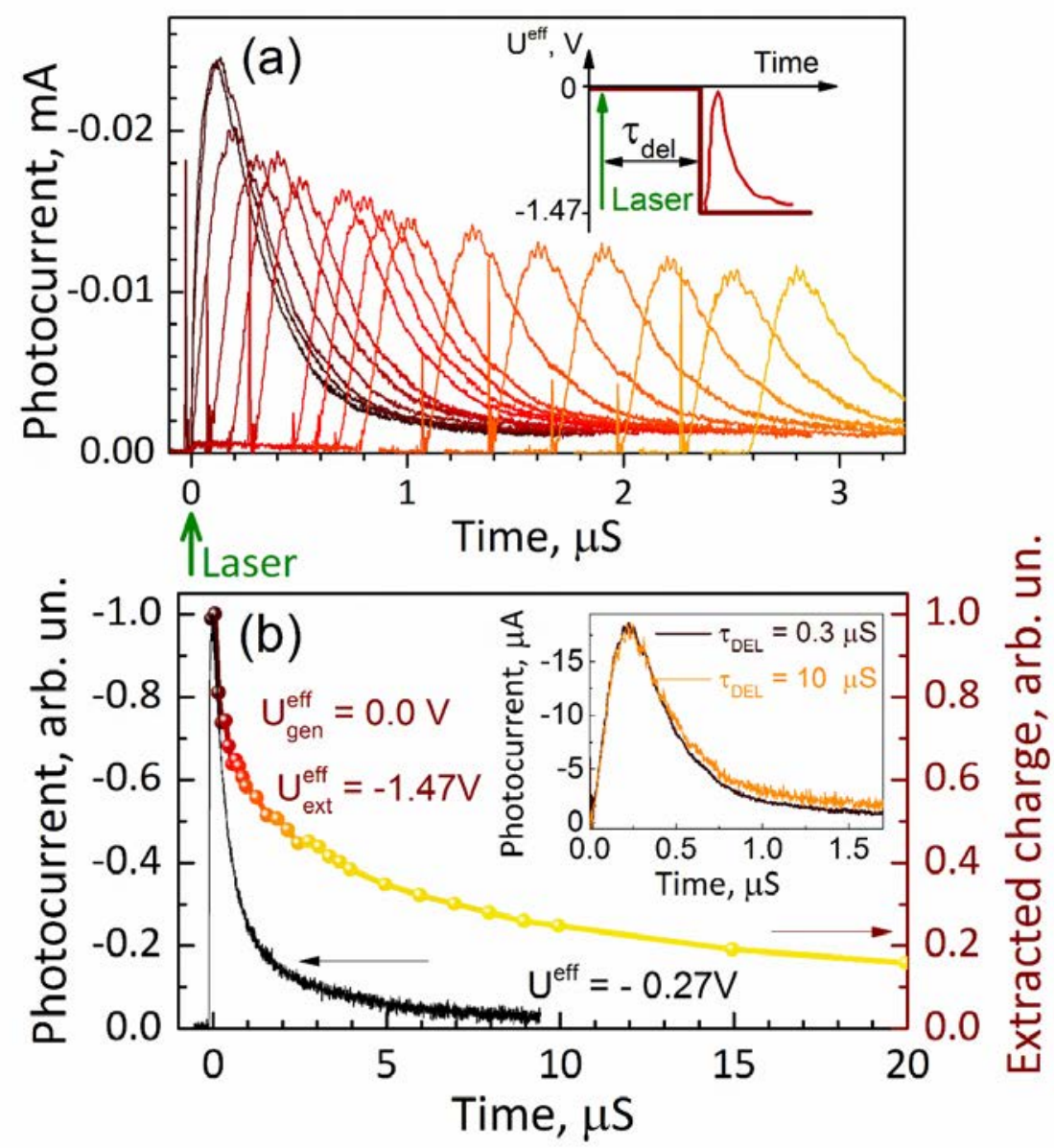




\section{WILEY-VCH}

Figure 2. a) Transient photocurrent created by a $-1.47 \mathrm{~V}$ effective collection voltage switched at different delay times after optical excitation under open-circuit conditions $\left(U_{\text {gen }}^{\text {eff }}=0\right)$. b) Normalized conventional photocurrent transient at a constant effective voltage $\mathrm{U}^{\text {eff }}=-0.27 \mathrm{~V}$ (black line) and the dependence of the total collected charge on the collection delay time corresponding to panel (a), i.e. for $U_{\text {gen }}^{\text {eff }}=0$ and $U_{\text {ext }}^{\text {eff }}=-1.47 \mathrm{~V}$ (red-yellow line with symbols). Measurements are taken on the as-cast device. The inset shows the normalized photocurrent transients at collection field switching delays of $0.3 \mu$ s and $10 \mu s$.

Figure 2a shows the dependence of the TDCF photocurrent kinetics on the collection delay time measured at close to zero effective generation voltage. The measurement process is schematically shown in the inset. These measurements correspond to open-circuit conditions when the built-in voltage was compensated by the applied voltage. Perfect compensation of the internal electric field to reach flatband is hardly possible due to band bending; nevertheless, the net field under these conditions is weak and is for simplicity defined as zero. As expected, the intensity of the photocurrent burst in Figure 2a decreases with delay time, which may be related to three processes. First, a decay of the charge carrier density due to recombination and, possibly, some diffusion to the electrodes; second, a decrease of the carrier mobility due to charges relaxing in the tail of the density of states (DOS) - this process could also be referred to as energetic trapping; third, a decay in the fraction of mobile charges due to charges 'getting stuck' in spatial traps.

Decrease in carrier mobility can be ruled out from the fact that the shape of the photocurrent burst does not change with the collection delay time, as shown in the inset of Figure 2b, indicating that carrier mobility does not change within the used collection delay time (otherwise the burst would broaden by slower carrier extraction). This is expected since charge carrier relaxation in the DOS and the associated mobility decay usually terminate on a sub-microsecond time scale. ${ }^{[6,19,20]}$ Consequently, the integrated areas of the current bursts that are plotted in the gradient curve with symbols in Figure $2 \mathrm{~b}$ correspond to the carrier densities that can be extracted from the device after the delay time, reflecting the charges that have not recombined or moved to electrodes, and did not get stuck in spatial traps. 


\section{WILEY-VCH}

The black line in Figure $2 \mathrm{~b}$ shows the conventional transient photocurrent response obtained at the same excitation intensity for a low, constant effective voltage of $-0.27 \mathrm{~V}$, when, according to the inset in Figure 1 (follow the dashed horizontal and vertical lines), no more than $40 \%$ of the photogenerated charge carriers were extracted (assuming all carriers are extracted at $U_{\mathrm{ext}}^{\text {eff }}=-1.47 \mathrm{~V}$ ). Thus, carrier extraction can only explain a minor fraction of the photocurrent drop at low constant voltage. This is confirmed by the fact that the conventional photocurrent decay (black curve in Figure $2 \mathrm{~b}$ ) reaches zero at $\sim 10 \mu \mathrm{s}$, whereas the TDCF measurements (red-yellow curve) show that there is still a significant amount of charge left in the sample after $10 \mu \mathrm{s}$. Since the TDCF data were taken under flatband conditions, they represent an upper limit to charge carrier recombination, that is, recombination in the conventional photocurrent experiment will be weaker, leaving more charges that could potentially be extracted. For these reasons, the photocurrent decay at constant voltage is only in part determined by recombination and extraction. Apparently, at small electric fields, a significant fraction of charge carriers gets trapped on a time scale of several microseconds.

On the other hand, the slow decay of the total extracted charge in the TDCF experiments indicates that the carrier trapping plays a relatively minor role in carrier extraction by delayed collection field (red-yellow symbols in panel b). Note that, in contrast to the conventional photocurrent experiment in Figure 2b, the generation field in the TDCF experiments is zero. The straightforward explanation could be that the about 5 times stronger collection field facilitates the release of trapped carriers. An alternative explanation would be that carriers get less trapped at the flatband conditions during the waiting time in TDFC experiment, when their motion is caused only by undirected diffusion, but do get trapped at finite electric fields, when they experience directed motion by drift and reach dead ends in the phase separated morphology, i.e. get stuck in spatial traps. This scenario bears strong resemblance to the findings on the small molecule:insulator blends discussed in the introduction. ${ }^{[15,16]}$ We also 


\section{WILEY-VCH}

note that charge motion under weak electric fields is of utmost relevance for device performance since the maximum power point of a solar cell is typically close to flatband.
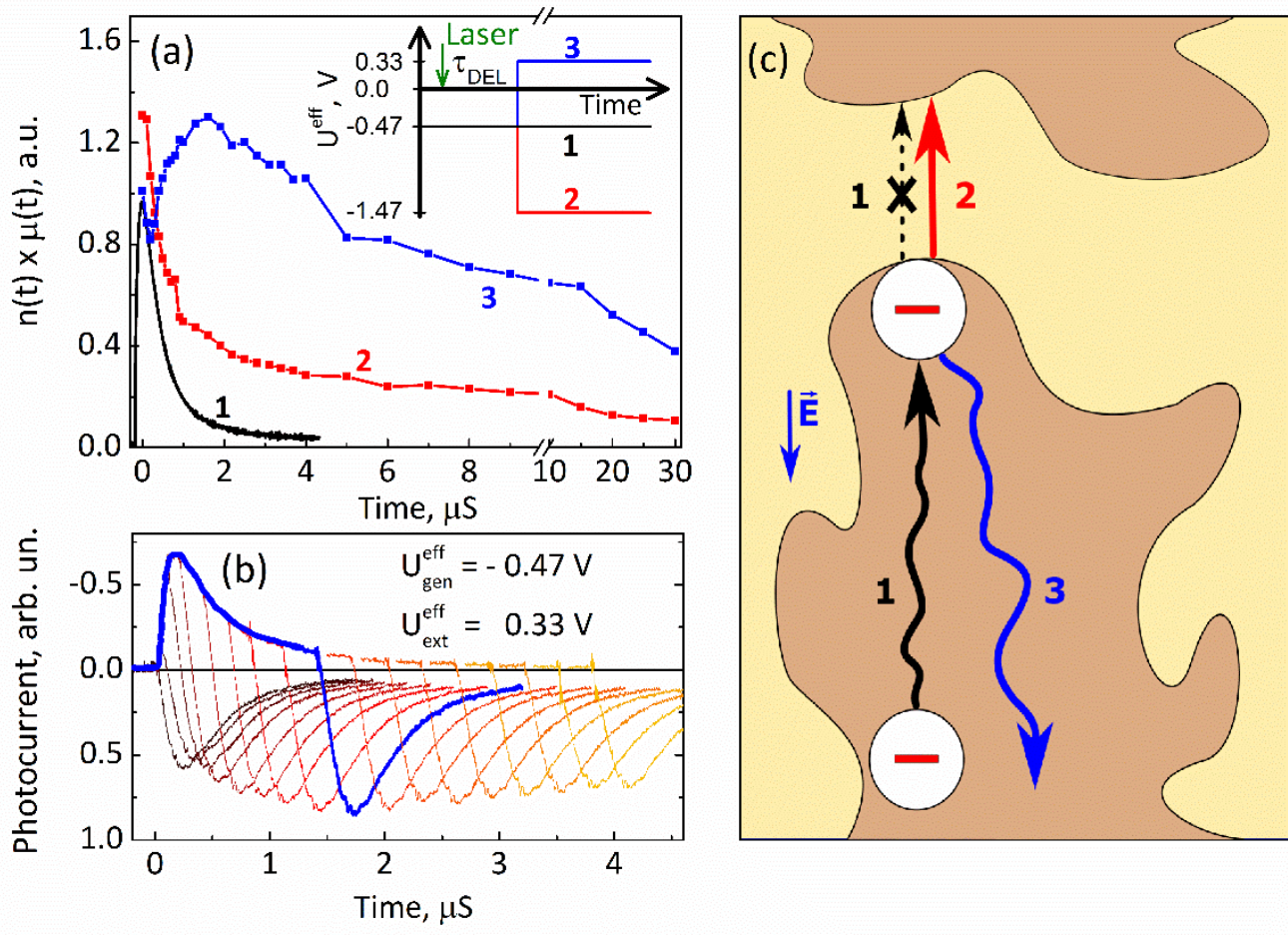

Figure 3. a) Photocurrent transient vs. time at constant field equal to the build-in field (black curve) and peak value of the TDCF photocurrent transient vs. collection delay time for different collection field strengths and directions as indicated in the inset (blue and red curves). b) Photocurrent transients at different collection delay times for a reversed collection field (blue curves in panel a), the thick blue line highlights one of the current transients. c) Illustration of the characteristic processes in the response to the different field profiles used in a). Measurements are taken on the as-cast device.

To invigorate our hypothesis about the importance of spatial traps in our all-polymer OPV devices, we have used a modified TDCF technique. We have used an effective generation voltage equal to the build-in potential ( $\mathrm{U}_{\text {gen }}^{\text {eff }}=-0.47 \mathrm{~V}$, zero applied bias) and different effective extraction voltages: a) equal to the generation voltage (degenerate TDCF), b) increased to $-1.47 \mathrm{~V}$ and c) reversed to $0.33 \mathrm{~V}$. These measurement regimes are schematically shown in the inset of Figure 3a. The main panel of Figure 3a presents the results. In contrast to Figure 2, we here present peak values of the transient photocurrent j(t) obtained after 


\section{WILEY-VCH}

switching on the collection field, divided by the electric field strength to get the product of the carrier density $\mathrm{n}(\mathrm{t})$ and mobility $\mu(\mathrm{t})$ from $j(t)=n(t) \mu(t) F$. Because of the sample capacitor recharging time, the photocurrent peak appears 250 ns after application of the collection voltage. However, this time is short in comparison with the carrier density decay time, so we may consider the $n(t) \mu(t)$ product at the photocurrent peak time identical for all three field profiles.

The differences between $n(t) \mu(t)$ values obtained for the different field profiles represent only differences in the fraction of mobile carriers: first, the total carrier densities were identical because of the equal pump intensities and equal generation fields and, second, relaxation phenomena are unimportant at the $\mu$ s time scales probed here (c.f. Figure $2 b$ inset). The black line shows $n(t) \mu(t)$ under constant applied voltage, the red line shows the same for an increased extraction field. In absence of spatial trapping, one would not expect any additional extraction upon increasing the (negative) extraction voltage after the constant voltage curve has dropped to zero. Instead, the increased field leads to the additional extraction of a significant number of photocreated charges, as witnessed by the red curve lying above the black one. The slow decay of this component is similar to the collection kinetics measured at zero generation field, c.f. Figure 2b. The presence of this additional, field-activated component is consistent with the above made conclusions, indicating the presence of spatially trapped charges that can survive tens of microseconds and that can be extracted by stronger electric fields.

If, as suggested above, charge carriers get stuck by drifting into dead ends that act as spatial traps, reversing the field should release them and give rise to a significant increase in the extracted charge. Figure 3b shows the photocurrent transients obtained with the reversed effective voltage at different collection delay times. As the thick blue curve, highlighting one of the current transients, shows, the field flipping increases the magnitude of the photocurrent drastically, even though the extraction field was slightly weaker than the generation field of 


\section{WILEY-VCH}

opposite direction. The increase is particularly strong at long delay times. The blue line in Figure 3a shows the delay time dependence of the peak values of the extracted photocurrent. The extracted photocurrent decays very slowly. Moreover, the photocurrent increases during the initial few $\mu$ s, i.e. precisely the time range for which the decay of the photocurrent at constant field (black curve) was attributed to spatial carrier trapping. These observations confirm the occurrence of spatial trapping of photocreated charges.

By comparing the peaks in the black and blue curves in Figure 3a, one can estimate the fraction of charges that get lost through spatial trapping on $0-2 \mu$ s timescales to be $\sim 30 \%$. This number is in excellent agreement with the $\sim 35 \%$ of the charges that were found to be bound in interfacial charge-transfer states and that recombine geminately on basis of a kinetic analysis of transient absorption spectroscopy measurements. ${ }^{[17]}$ We should point out that trapping itself is not a loss channel; the actual loss channel is the recombination of charges that do not manage to escape from their countercharge because of spatial trapping. Along the same lines, (spatial) trapping occurring at longer time and length scales will no longer lead to increased geminate recombination but will contribute to losses through bimolecular recombination.

The drawing in Figure 3c illustrates our interpretation of the carrier extraction experiments. A fraction of the carriers drifting in one direction reach a dead end of a particular blend domain (process 1) and discontinue their drift. Occasionally they can overcome the barrier and continue to drift towards the extracting electrode, causing the weak and slow photocurrent component observed at constant applied voltage. This process (2) is assisted by the electric field. While they are trapped, charges are susceptible to (predominantly geminate) recombination, explaining the poor fill factor of the as-cast devices. When a reversed collection field is applied, charge carriers are detrapped by moving in the opposite direction (process 3). 


\section{WILEY-VCH}

The ns- $\mu$ s timescale of the experiments in Figure 3 may appear long compared to the ps-ns timescales that are commonly associated with geminate recombination. However, conventional geminate recombination is associated with charges not escaping from the exciton or CT state, i.e. with electron-hole pairs that have substantial wavefunction overlap. In the present case, we are monitoring charges that did escape the exciton/CT state in first instance but get stuck afterwards. When these charge pairs eventually reform a (CT) exciton and recombine, the kinetics are still first order, but occur on extended time scales associated with the re-forming process. We note that the ns- $\mu$ s timescales in Fig. 3 are consistent with the (geminate) recombination timescales observed in Ref. 17.

a) TQ1:N2200 annealed

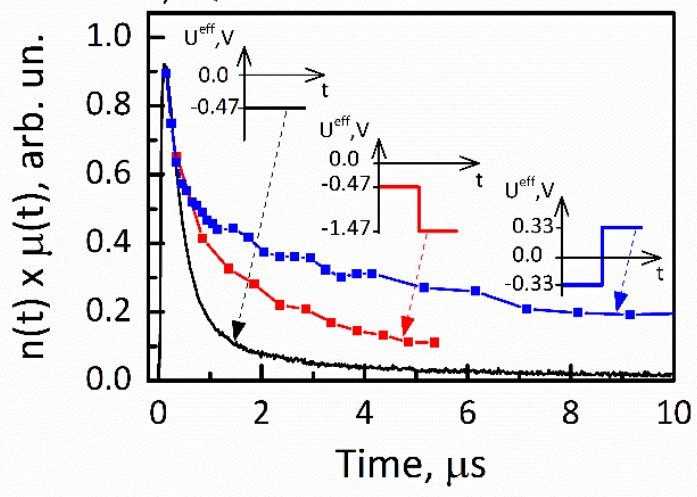

b) TQ1:PC71BM

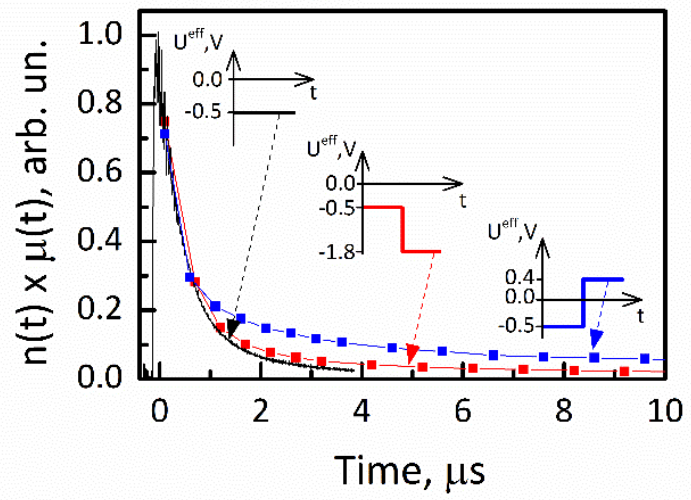

Figure 4. Photocurrent transient vs. time at constant field equal to the build-in field (black curve) and peak values of the TDCF photocurrent transient vs. collection delay time for different collection field strengths and directions as indicated in the inset (blue and red curves). Measurements are taken on the a) TQ1:N2200 annealed and b) TQ1:PC71BM devices.

According to the suggested interpretation, the spatial trapping effect should be a very sensitive probe of the blend morphology. We have therefore performed the same investigations of annealed samples. Annealing the investigated TQ1/N2200 solar cells roughly doubles their power conversion efficiency to $\sim 3.15 \% .{ }^{[18]}$ Figure 4 a shows the TDCF investigation results for different applied field profiles. Most strikingly, the different peak values of the three curves that are found for the as-cast sample (Figure 3) are absent within 


\section{WILEY-VCH}

experimental resolution, indicating that the short-range spatial trapping, and the associated geminate recombination loss, have largely vanished. This provides a transparent explanation of the previously puzzling observation that the geminate recombination loss dropped from $\sim 35 \%$ to $\sim 7 \%$ upon annealing while unaffecting long-range transport. ${ }^{[17]}$ Apparently, annealing removes spatial traps that hamper short-range charge motion required to escape geminate recombination. In view of the nm length scales involved in geminate recombination, these dead ends are most likely associated with individual polymer chain ends.

At longer delay times, the (blue) curve corresponding to opposite generation and extraction fields lies above the (red) curve corresponding to generation and extraction fields of equal polarity. Since the plotted data have been corrected for dark currents, the difference cannot be attributed to the difference in forward and reverse injection currents. Instead, they point towards a large fraction of charges surviving in the active layer while being spatially trapped. This indicates that even after annealing, spatial traps continue to play a role in this allpolymer BHJ. As mentioned above, these trapped charges are predominantly exposed to bimolecular recombination. Although the associated rate constant in as-cast and annealed BHJ is roughly the same (essentially because the mobility is the same), the loss to bimolecular recombination will be largest in the as-cast films because of a starker spatial trapping, and presumably due to larger trap densities. Combined with the geminate loss discussed above, this explains the roughly factor of two difference in power conversion efficiency between the two devices.

Potential minima that can occur due to band bending near contacts at near-flatband conditions could in principle also act as 'spatial traps', although it would be hard to rationalize why they would predominantly give rise to geminate recombination. Nevertheless, we confirmed that such potential wells are not at work in our experiments by comparing photocurrent transients at opposite (net) fields of equal magnitude. The photocurrent transients were identical and are presented in the SI. Since processes leading to band bending in the vicinity of contacts like 


\section{WILEY-VCH}

Schottky barrier formation, ${ }^{[21]}$ thermal diffusion of charges from the contacts ${ }^{[22]}$ or minority carrier accumulation ${ }^{[23]}$ are asymmetric, the complete equivalence of the obtained current transients rules out such scenarios.

In principle, also an effective medium with only diagonal disorder can give rise to dead ends. Diagonal disorder here refers to energetic disorder in the site energies; spatial traps would be considered a manifestation of off-diagonal disorder, i.e. a variation in the tunneling barrier between neighboring sites. In the case of diagonal (on-site) disorder, dead ends are sites that are connected to the percolating cluster but only from one side. Such 'energetic dead ends' might give rise to the same behavior as observed in our experiments - a charge can for example move into (a series of) sites by 'easy' hops along the direction of the applied field but then run into a 'difficult' hop and get stuck. To rule out this scenario, we ran explicit kinetic Monte Carlo simulations of the TDCF experiment (see SI). The results clearly did not match the experimental observations indicating that the diagonal disorder alone cannot explain the presence of effective spatial traps.

Since we associate the spatial trapping with the specific properties of an all-polymer BHJ, we performed a similar investigation as above for a solar cell based on the same donor material TQ1, blended in a bulk heterojunction with $\mathrm{PC}_{71} \mathrm{BM}$ as acceptor in a mass ratio of 1:2.5. These samples showed only small differences between the transient photocurrent at constant applied field and the carrier concentration decays measured with the TDCF technique (see Figure 4b). Nevertheless, the carrier concentration extracted with the reversed effective voltage was slightly larger than with increased effective voltage, which indicates that spatial trapping still takes place in these devices. However, the spatial trapping is significantly less prominent than in both the pristine and annealed TQ1/N2200 blends, in line with the anticipated behavior of polymer:small molecule BHJ discussed in the introduction. Also, in view of the stoichiometry of our samples the limited spatial trapping is not particularly surprising. However, in previous work ${ }^{[24]}$ we found a strong decrease of the electron drift rate 


\section{WILEY-VCH}

upon decreasing the PCBM stoichiometric ratio in blends below $~ 50 \%$ by weight, which might point towards spatial trapping in dead ends of fullerene-rich domains under these conditions.

\section{Conclusion}

We have investigated charge carrier motion in an archetypical all-polymer organic solar cell by a combination of carrier extraction experiments and numerical modeling. We find that in the as-cast active layer, the bulk heterojunction morphology contains a significant concentration of spatial traps that we tentatively associate with individual polymer chain ends. The presence of these spatial traps manifests in a suppressed yield of free charge generation due to one of the charges in the splitting CT pair 'getting stuck' close to its countercharge, leading to enhanced geminate recombination. Likewise, spatial trapping of otherwise free charges occurs and leads to enhanced bimolecular recombination. The effects of the offdiagonal disorder, i.e. the spatial traps, occur in addition to those of the diagonal (on-site) disorder that we previously identified as the cause for highly dispersive, non-equilibrium charge motion. While these findings highlight a morphological problem that seems most likely to arise in all-polymer BHJ (in BHJ based on the same polymer donor with a small molecule (PCBM) acceptor spatial trapping is much weaker) they also show that the number of spatial traps can, at least in the present case, be drastically reduced by thermal annealing.

\section{Experimental Section}

All-polymer solar cells of inverted geometry were prepared from poly[2,3-bis-(3octyloxyphenyl)quinoxaline-5,8-diyl-alt-thiophene-2,5-diyl] (TQ1), which served as an electron donor, and poly\{[N,N'-bis(2-octyldodecyl)naphthalene-1,4,5,8-bis(dicarboximide)2,6-diyl]-alt-5,5'-(2,2'-bithiophene)\} (known as P(NDI2OD-T2), PNDI-2T or N2200) acting as electron acceptor. ${ }^{[25,26]}$ BHJ active layers of TQ1:N2200 (2:1 ratio by weight) were spincoated from a $9 \mathrm{~g} / \mathrm{L}$ (total) chloroform solution on $\mathrm{ZnO} / \mathrm{ITO} /$ glass substrates, yielding an 


\section{WILEY-VCH}

active layer thickness of $85 \mathrm{~nm}$. $\mathrm{A} \mathrm{MoO}_{3} / \mathrm{Al}$ top electrode was thermally evaporated through a shadow mask. Annealed samples were prepared by heating the substrate with the active layer for $10 \mathrm{~min}$ at $120{ }^{\circ} \mathrm{C}$ before top electrode deposition. Samples were fabricated inside an $\mathrm{N}_{2}$-filled glovebox and encapsulated with epoxy glue. Detailed information on OPV device preparation can be found elsewhere. ${ }^{[18]}$ The annealed solar cells had a PCE of $\sim 3 \%$, while PCE of as-spun samples was roughly twice lower.

Bulk heterojunction polymer-fullerene inverted solar cells were fabricated on pre-cleaned glass substrates. $\mathrm{Ti}(2 \mathrm{~nm}) / \operatorname{Al}(80 \mathrm{~nm}) / \mathrm{Ti}(2.5 \mathrm{~nm})$ contact (cathode) was first thermally evaporated on glass under high vacuum conditions. The samples were exposed to ambient for 1 minute to form TiOx. PFPA-1 in toluene with a concentration of $2 \mathrm{mg} \mathrm{mL}^{-1}$ was spin coated on top of the TiOx surface at $1000 \mathrm{rpm}$ for 60s (in the glovebox), followed by rinsing with odichlorobenzene (oDCB) to create a monolayer of PFPA-1. ${ }^{[27]}[6,6]-p h e n y l C_{71}$ butyric acid methyl ester ( $\left.\mathrm{PC}_{71} \mathrm{BM}\right)$ was selected as an electron acceptor while TQ1 as an electron donor. The TQ1:PC ${ }_{71} \mathrm{BM}$ active layer (1:2.5 weight ratio, $20 \mathrm{gL}^{-1}$ in oDCB) was spin coated on the PFPA-1 monolayer at 500 rpm for 60s and dried at 3000 rpm for 30s. PEDOT:PSS PH1000 mixed with 5\% dimethyl sulfoxide (DMSO) and 0.5\% surfactant (Zonyl FS 300) was spincoated on the active layer in ambient with $1000 \mathrm{rpm}$ for $60 \mathrm{~s}$. The samples were annealed at $60{ }^{\circ} \mathrm{C}$ for 1 minute before encapsulation in the glovebox. The thickness of the active layer was around $80 \mathrm{~nm}$ as measured by a Dektak surface profilometer.

Transient photocurrent investigations were performed with an Agilent Technologies DS05054A oscilloscope and a Tektronix AFG 3101 function generator. Samples were illuminated by sub-nanosecond $532 \mathrm{~nm}$ laser pulses with a $5 \mathrm{~Hz}$ repetition rate. Transient photocurrent was measured using the $50 \Omega$ input resistor of the oscilloscope. The time resolution of the current measurements was about 100-200 ns, mainly determined by the oscilloscope input resistance and the sample capacitance of $\sim 1.5 \mathrm{nF}$. In integral-mode measurements, the $1 \mathrm{M} \Omega$ oscilloscope input resistor was used instead, while the sample and 


\section{WILEY-VCH}

the oscilloscope capacitances served as the integrating capacitor. The time resolution of integral-mode measurements is about 100 ns, limited by the capacitance and inductivity of the electrical circuit.

Time-delayed collection field (TDCF) ${ }^{[28]}$ investigations were performed with the same experimental setup as used in transient photocurrent measurements. An adjustable voltage was applied to the sample during the optical excitation (generation voltage). Following a variable time delay, the collection voltage was switched on. The voltage generator was triggered by the optical excitation pulse. To compensate the triggering delay of the voltage generator for the experiments in Figures 2-4, the excitation pulse passed a $100 \mathrm{~m}$ long optical fiber, acting as a 2300 ns delay line, before reaching the sample. This allowed to reduce the shortest effective delay time to 10 ns. The shown charge extraction kinetics correspond to the difference between the current transients with and without optical excitation.

\section{Supporting Information}

Supporting Information is available from the Wiley Online Library or from the author.

\section{Acknowledgements}

This research was partly funded by the European Social Fund according to the activity 'Improvement of researchers' qualification by implementing world-class R\&D projects' of Measure No. 09.3.3-LMT-K-712-01-0031

Received: ((will be filled in by the editorial staff))

Revised: ((will be filled in by the editorial staff)) Published online: ((will be filled in by the editorial staff))

\section{References}

[1] R. Mauer, I. A. Howard, F. Laquai, J. Phys. Chem. Lett. 2010, 1, 3500.

[2] C. Deibel, V. Dyakonov, Rep. Prog. Phys. 2010, 73, 096401.

[3] L. J. A. Koster, Phys. Rev. B 2010, 81, 205318. 


\section{WILEY-VCH}

[4] S. H. Park, A. Roy, S. Beaupré, S. Cho, N. Coates, J. S. Moon, D. Moses, M. Leclerc, K. Lee, A. J. Heeger, Nat. Photonics 2009, 3, 297.

[5] L. J. A. Koster, E. C. P. Smits, V. D. Mihailetchi, P. W. M. Blom, Phys Rev B 2005, 72, 085205.

[6] A. Melianas, F. Etzold, T. J. Savenije, F. Laquai, O. Inganäs, M. Kemerink, Nat. Commun. 2015, 6, 8778.

[7] F. Symalla, P. Friederich, A. Massé, V. Meded, R. Coehoorn, P. Bobbert, W. Wenzel, Phys. Rev. Lett. 2016, 117, 276803.

[8] A. Massé, P. Friederich, F. Symalla, F. Liu, V. Meded, R. Coehoorn, W. Wenzel, P. A. Bobbert, Phys. Rev. B 2017, 95, 115204.

[9] A. Melianas, V. Pranculis, D. Spoltore, J. Benduhn, O. Inganäs, V. Gulbinas, K. Vandewal, M. Kemerink, Adv. Energy Mater. n/a.

[10] H. Abdalla, G. Zuo, M. Kemerink, Phys Rev B in press.

[11] W. Zhao, S. Li, H. Yao, S. Zhang, Y. Zhang, B. Yang, J. Hou, J. Am. Chem. Soc. 2017, 139, 7148.

[12] Y. Li, J.-D. Lin, X. Che, Y. Qu, F. Liu, L.-S. Liao, S. R. Forrest, J. Am. Chem. Soc. 2017, 139, 17114.

[13] Y. Lin, F. Zhao, Y. Wu, K. Chen, Y. Xia, G. Li, S. K. K. Prasad, J. Zhu, L. Huo, H. Bin, Z.-G. Zhang, X. Guo, M. Zhang, Y. Sun, F. Gao, Z. Wei, W. Ma, C. Wang, J. Hodgkiss, Z. Bo, O. Inganäs, Y. Li, X. Zhan, Adv. Mater. 2017, 29, 1604155.

[14] T. Kim, J. Choi, H. J. Kim, W. Lee, B. J. Kim, Macromolecules 2017, 50, 6861.

[15] P. M. Borsenberger, L. Pautmeier, H. Bässler, J. Chem. Phys. 1991, 94, 5447.

[16] D. Hertel, H. Bässler, ChemPhysChem 2008, 9, 666.

[17] S. Karuthedath, A. Melianas, Z. Kan, V. Pranculis, M. Wohlfahrt, J. I. Khan, J. Gorenflot, Y. Xia, O. Inganäs, V. Gulbinas, M. Kemerink, F. Laquai, Mater. Chem. A, 2018, Advance Article . 


\section{WILEY-VCH}

[18] Y. Xia, C. Musumeci, J. Bergqvist, W. Ma, F. Gao, Z. Tang, S. Bai, Y. Jin, C. Zhu, R. Kroon, C. Wang, M. R. Andersson, L. Hou, O. Inganäs, E. Wang, J. Mater. Chem. A 2016, 4, 3835.

[19] A. Devižis, A. Serbenta, K. Meerholz, D. Hertel, V. Gulbinas, Phys. Rev. Lett. 2009, 103, 027404.

[20] A. Melianas, V. Pranculis, Y. Xia, N. Felekidis, O. Inganäs, V. Gulbinas, M. Kemerink, Adv. Energy Mater. 2017, 7, 1602143.

[21] I. Lange, J. C. Blakesley, J. Frisch, A. Vollmer, N. Koch, D. Neher, Phys. Rev. Lett. 2011, 106.

[22] M. Kemerink, J. M. Kramer, H. H. P. Gommans, R. A. J. Janssen, Appl. Phys. Lett. 2006, 88, 192108.

[23] G. Garcia-Belmonte, A. Munar, E. M. Barea, J. Bisquert, I. Ugarte, R. Pacios, Org. Electron. 2008, 9, 847.

[24] V. Pranculis, Y. Infahsaeng, Z. Tang, A. Devižis, D. A. Vithanage, C. S. Ponseca, O. Inganäs, A. P. Yartsev, V. Gulbinas, V. Sundström, J. Am. Chem. Soc. 2014, 136, 11331.

[25] E. Wang, J. Bergqvist, K. Vandewal, Z. Ma, L. Hou, A. Lundin, S. Himmelberger, A. Salleo, C. Müller, O. Inganäs, F. Zhang, M. R. Andersson, Adv. Energy Mater. 2013, 3, 806.

[26] D. Mori, H. Benten, I. Okada, H. Ohkita, S. Ito, Adv. Energy Mater. 2014, 4, n/a.

[27] Z. Tang, L. M. Andersson, Z. George, K. Vandewal, K. Tvingstedt, P. Heriksson, R. Kroon, M. R. Andersson, O. Inganäs, Adv. Mater. 2012, 24, 554.

[28] J. Kniepert, M. Schubert, J. C. Blakesley, D. Neher, J. Phys. Chem. Lett. 2011, 2, 700. 


\section{WILEY-VCH}

Archetypical all-polymer bulk heterojunction solar cells contain a significant concentration of spatial traps associated with the dead ends of polymer chains. The spatial trapping significantly impedes the carrier extraction. The trapping effect weakens by annealing the device, and is much weaker in devices based on the same donor polymer and a fullerene acceptor.

\section{Organic photovoltaic, all-polymer solar cell, spatial traps, carrier extraction}

Rokas Jasiūnas, Armantas Melianas, Yuxin Xia, Nikolaos Felekidis, Vidmantas Gulbinas* and Martijn Kemerink

Dead ends limit charge carrier extraction from all-polymer bulk heterojunction solar cells

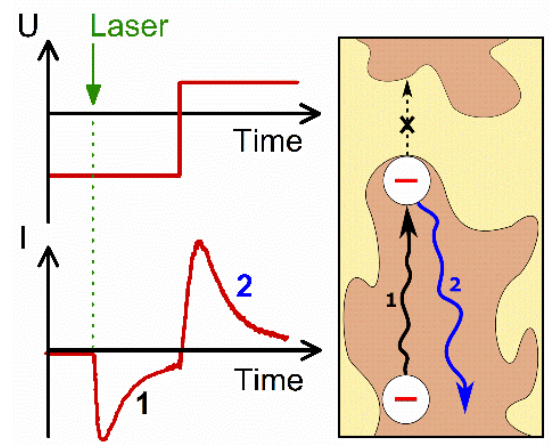




\section{WILEY-VCH}

Copyright WILEY-VCH Verlag GmbH \& Co. KGaA, 69469 Weinheim, Germany, 2016.

\section{Supporting Information}

\section{Dead ends limit charge carrier extraction from all-polymer bulk heterojunction solar cells}

Rokas Jasiūnas*, Armantas Melianas, Yuxin Xia, Nikolaos Felekidis, Vidmantas Gulbinas and Martijn Kemerink

\section{Transient photocurrent for different effective voltage polarities}

Figure S1 shows the transient photocurrent kinetics measured for as spun and annealed samples at steady-state voltages of equal effective values but of opposite polarities. Very similar kinetics show that the internal electric field for both voltage polarities was identical only of different direction. It indicates that the role of band bending near contacts in carrier extraction measurements was weak or absent.

(a) As-spun

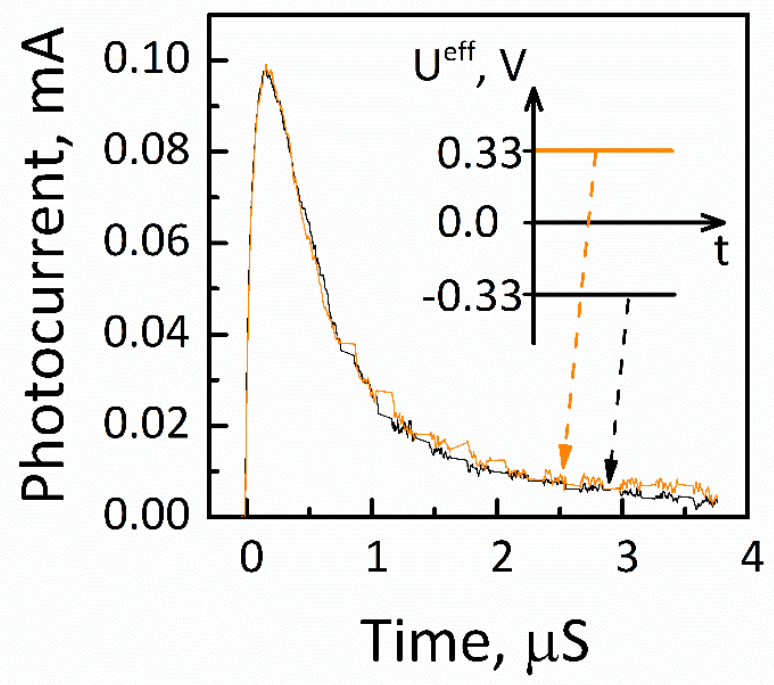

(b) Annealed

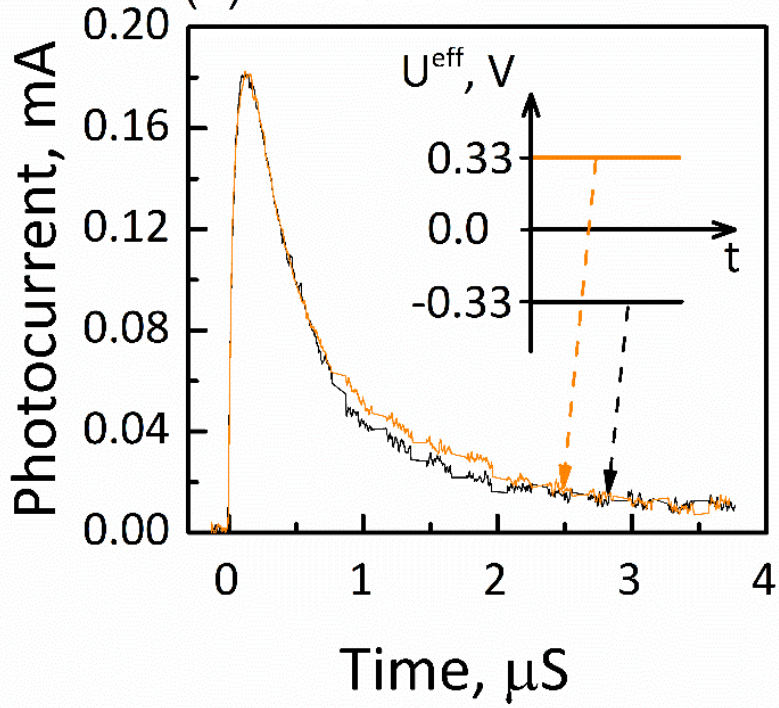

Figure S1. Transient photocurrent kinetics at constant positive and negative effective voltages of $\pm 0,33 \mathrm{~V}$ (degenerate TDCF regime). Plots a) and b) show results for the as-spun and annealed samples respectively.

\section{Influence of diagonal disorder}

Sites that are connected to the percolating cluster but only from one side may form 'energetic dead ends' that might give rise to the same behavior as observed in our experiments - a charge can e.g. move into (a series of) sites by 'easy' hops along the direction of the applied 


\section{WILEY-VCH}

field but then run into a 'difficult' hop and get stuck. To rule out this scenario, we ran explicit kinetic Monte Carlo simulations of the TDCF experiment. The used parameters were taken from previous work on related samples. We have previously shown that we can describe both ultrafast extraction experiments and steady-state transport. [S1] Unfortunately, these simulations are by the nature of the experiment extremely prone to numerical noise. Nevertheless, the results in Figure S2 do not clearly match the experimental observations. First, reversing the field 1-5 $\mu$ s after the (simulated) light pulse does not lead to a larger amount of extracted charge, as visible from the similar peak in the photocurrent transient with respect to the base line decay (thick black line); see e.g. the peaks just after 1 and $2 \mu \mathrm{s}$. In addition, reversing the field at longer delays does not release a significant reservoir of trapped charges, as seen from the absence of a significant response upon switching at $5 \mu$ s, c.f. red and blue curves. This picture is corroborated by the time dependency of the number of charges in the (simulated) device (not shown).
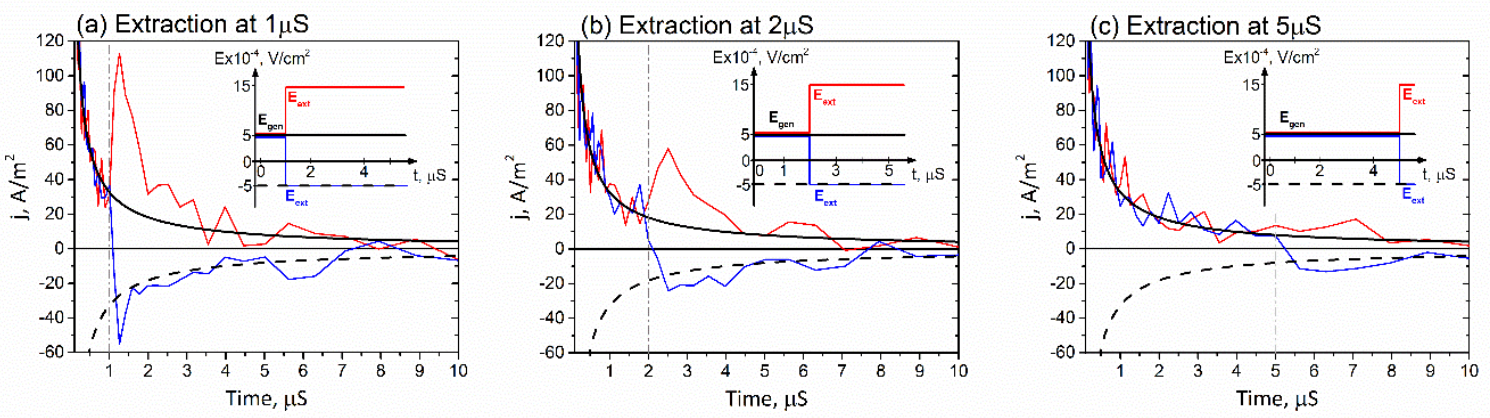

Figure S2. Calculated TDCF response for an effective medium with diagonal energetic disorder. $\mathrm{E}_{\text {gen }}=5 \times 10^{4} \mathrm{~V} / \mathrm{cm}, \mathrm{E}_{\text {ext }}=-5 \times 10^{4} \mathrm{~V} / \mathrm{cm}$ (blue lines), $\mathrm{E}_{\text {ext }}=15 \times 10^{4} \mathrm{~V} / \mathrm{cm}$ (red lines) or $\mathrm{E}_{\text {ext }}=\mathrm{E}_{\text {gen }}$ (black lines); The field is switched in 1 ns at $1 \mu \mathrm{s}, 2 \mu \mathrm{s}$ and $5 \mu$ s (panels a, $\mathrm{b}$ and c respectively) after populating a small fraction $\left(10^{-3}\right)$ of a $20 \times 20 \times 47$ box with excitons at $\mathrm{t}=0$. Results have been averaged over 3000 random configurations per trace. Other parameters are as used in our previous work on the same material system. ${ }^{[\mathrm{S} 1]}$

Hence, the simulations in Figure S2 show that our experimental findings cannot be attributed to diagonal disorder. We should, however, stress that this does not imply that the dispersive motion of photocreated charges that we previously attributed to energetic relaxation in the DOS must now be attributed to spatial disorder. Instead, the off-diagonal disorder that is found here comes atop the diagonal one. We can, however, not exclude that ignoring the former in our model will have affected the parameter values we found in the case of allpolymer blends in ref. S1, where the annealed TQ1:N2200 sample was investigated. The reason the off-diagonal disorder could be singled out in the present experiments is because these are performed on time scales at which energetic relaxation/trapping is close to complete.

\section{References}

[S1] A. Melianas, V. Pranculis, Y. Xia, N. Felekidis, O. Inganäs, V. Gulbinas, M. Kemerink, Adv. Energy Mater. 2017, 7, 1602143. 